\title{
Research and Design of Photoelectric Solar Tracking System
}

\author{
Tianhua Li, Deqing Xiang \\ Department of Physical and Electrical Engineering, Zunyi Normal College, Zunyi, Guizhou, 563002 \\ Ithyy@163.com
}

Keywords: photosensitive resistance; solar ray; metering tube

\begin{abstract}
In this paper, a set of solar tracking system based on the photosensitive resistance has been studied and designed from the perspectives of practicality, economy and reliability. The device is simple and reliable. The sensitivity is high and the cost is low. It is suitable for the solar tracking of various solar energy utilization devices such as the medium and small solar light collection systems, the solar power systems, the solar heating systems and some others.
\end{abstract}

\section{Introduction}

As a clean and non-polluting energy, the solar energy is inexhaustible. It not only refers to the energy supplement badly needed in the near term, but also the basis of future energy structure. As for the solar energy resources, the density of sunlight is low and the interval of exposure time as well as the spatial distribution is constantly changing. Currently, most of the solar concentrators are fixed. However, the direction and the intensity of lights are ever-changing. Then the solar energy resources are not fully utilized and the efficiency will be low. Therefore, it is required to adopt the photosensitive resistance to track the sun so as to make the lighting surface of system perpendicular to the incident directions of sun rays. Afterwards, more input radiation can be intercepted in the limited use area and the maximum absorption state of solar energy can be achieved. Then the use efficiency of solar energy will be improved and the application value of solar energy system will be increased ${ }^{[1]}$.

\section{The relationship between the positions of earth and sun}

The amount of sun lights collected by the sun light collection system depends on the scattering and the absorption of atmosphere for the lights. The sun light collection system can only collect the sun lights with direct radiation. As the radiation energy of scattering is very few, so it is important to collect the sun lights with direct radiation as much as possible. The sun lights with direct radiation can be collected to the maximum extent through tracking the movement directions of sun. As for the sun light collection system located at a position on the earth, the angle between the sun and its position at a certain time can be represented by the solar elevation angle $a_{s}$ and the sun azimuth angle $y_{s}$. Both of them are defined in the horizon coordinate system. The horizon coordinate system provides the observation point $\mathrm{O}$ which is also the point where the solar panels are located as the coordinate origin. The vertical direction of horizontal plane is Z-axis, the south direction of geography is X-axis and the east direction of geography is $\mathrm{Y}$-axis (shown in Figure 1). The sun elevation angle $\mathrm{a}_{\mathrm{s}}$ is the included angle between the local level and the sight vector from the observation point $\mathrm{O}$ to the sun $\mathrm{S}$, whose numeric area is between $0^{\circ} \sim 90^{\circ}$. The sun azimuth $Y_{\mathrm{s}}$ is the included angle between the projection of this sight vector on the local horizontal planes and the geographical south direction. At the same time, the due south is zero, the west direction is positive and the east direction is negative ${ }^{[2]}$. 


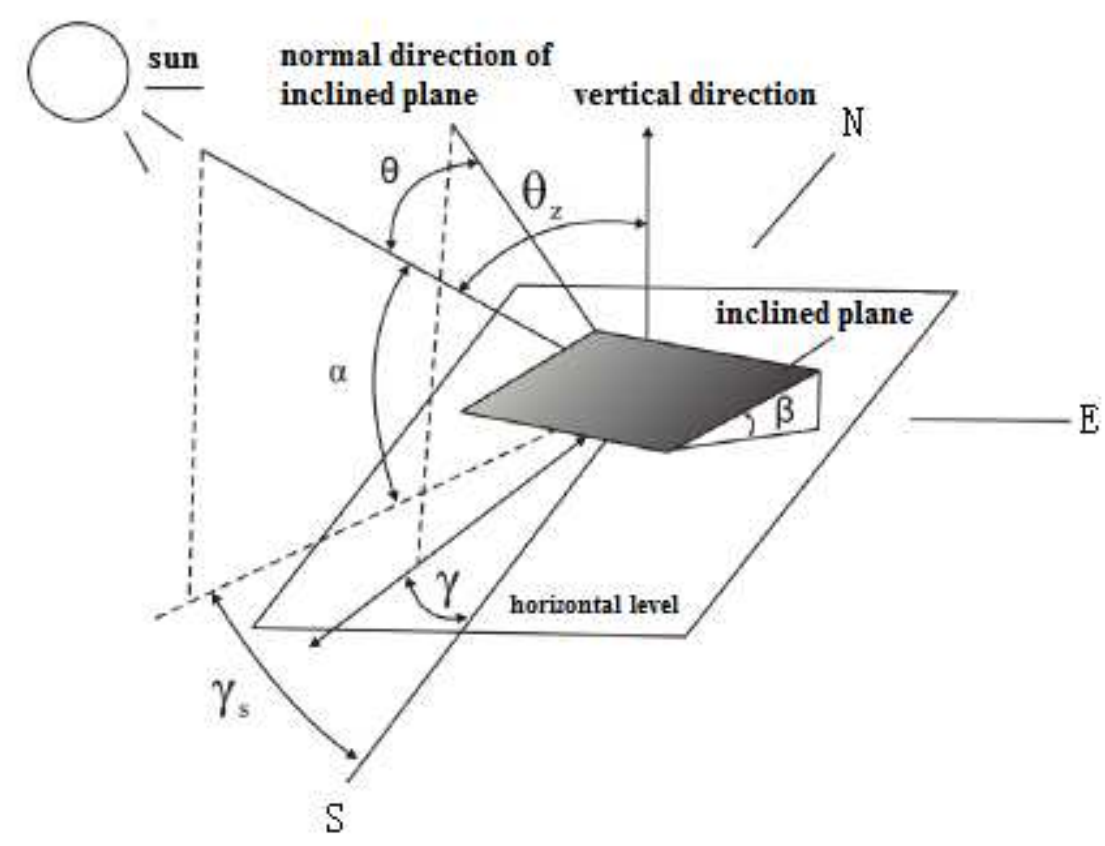

Figure 1 The relationship between the solar radiation angle and the inclined plane angle

The angle between the sun lights and the face normal of lighting components is called the incident angle $\theta$ of sun lights. The sun lights can be divided into two vectors. One vector is perpendicular to the lighting plane and another one is parallel with the lighting plane. Only the radiation of former vector can be intercepted by the lighting planes. Thus, it will be better if the incident angle $\theta$ is as small as possible in the practice. Then the tracking can be achieved. The azimuth angle of lighting plane is yand the face normal of lighting components has a projection on the ground. The included angle between this projection line and the south direction refers to the azimuth angle of lighting components. The inclined angle of the lighting components is $\beta$. The included angle between the lighting component plane and the horizontal plane is considered as the inclined angle of lighting components. The amount of solar radiation obtained by the lighting components mainly depends on the solar incident angle $\theta$. $\theta$ is the function of the sum of the solar declination angle $\delta$, the solar hour angle $\omega$, the geographic latitude $\varphi$, the inclined angle $\beta$ of lighting components and the azimuth angleyof lighting components. Therefore, when $\delta$, wand pare determined, the value of the inclined angle $\beta$ and the azimuth angleyof lighting components will determine the direct solar injection angle $\theta$. When the value of the inclined angle and the azimuth angle of lighting components is appropriately controlled, the incident angle $\theta$ of the sun lights will be close to $0^{\circ}$. And then the sun lights can be collected to the maximum extent ${ }^{[3]}$. 


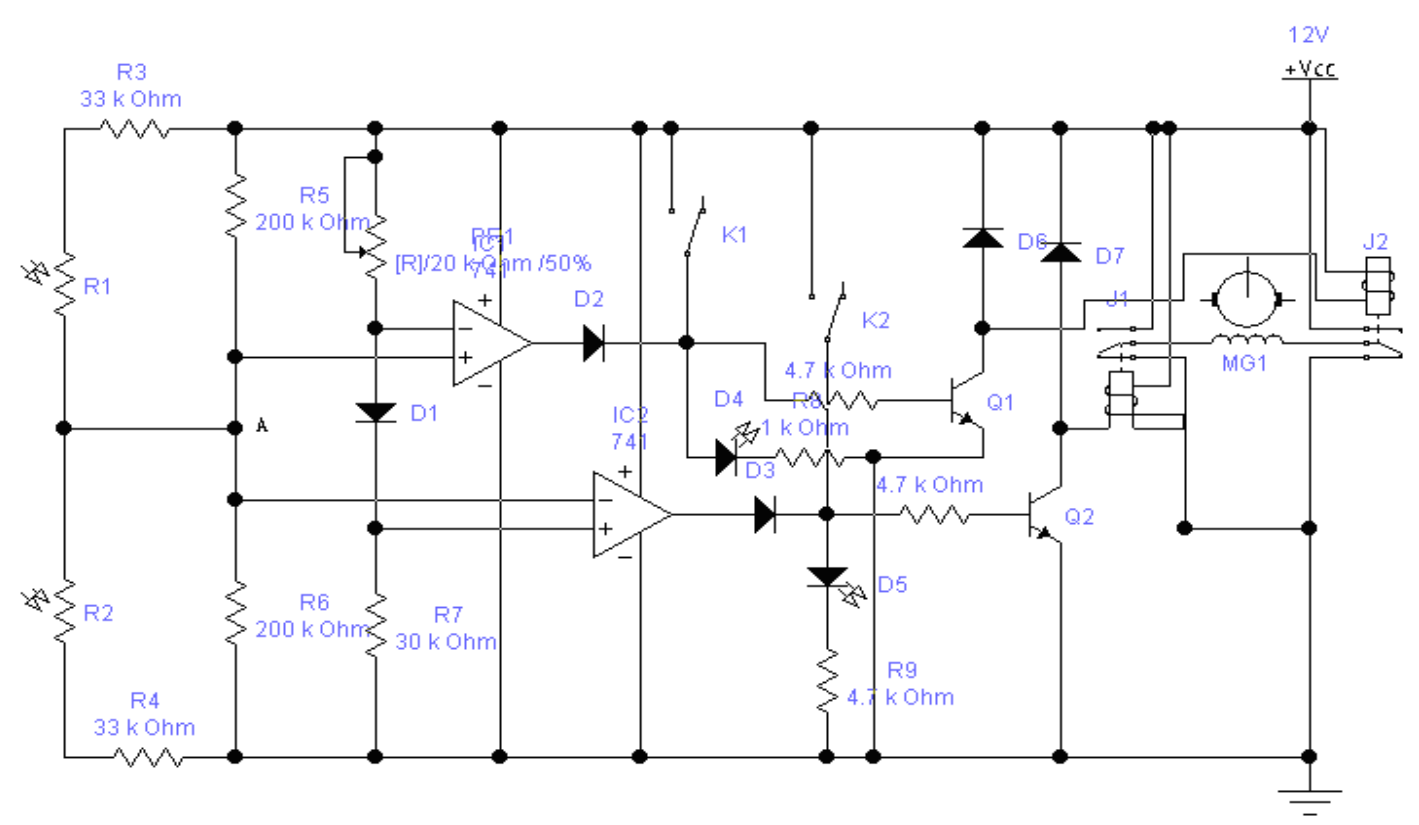

Figure 2 The circuit diagram of the sun light tracking system

\section{The tracking system}

As the position of the sun is located within an area of sky, so the dual-axis tracking should be adopted to track the sun in this paper. The dual-axis tracking can be the horizon coordinate system of observer or the hour angle coordinate system. No matter which system is employed, the two motors are required to adjust the position of condensers through their own sensing devices. Then the axis will point to the sun. As the support organization of the condenser of horizon coordinate system is relatively simple and the tracking is stable and reliable, so it is always adopted in engineering. At present, the methods to track the sun can be divided into the sun motion trajectory tracking, the optical tracking and the combination of sun motion trajectory tracking and optical tracking.

According to the principle that the resistance value of photosensitive resistor will be changing under illumination, two same photosensitive resistors have been placed in the probe head in this paper. If the sun lights vertically irradiate the probe head, the light intensity received by the two photosensitive resistors will be equal. So their resistance values are the same and then the motor does not rotate. When there is an included angle between the direction of sun lights and the vertical direction of probe head, the resistance value of photosensitive resistor which receives more light intensity will be decreased. Then the motor won't be driven to rotate until the light intensity of the two photosensitive resistors is equal. The advantage refers that the control is more accurate and the circuit is relatively easy to be implemented.

The circuit diagram of the tracking system (shown in Figure 2) is composed by the operational amplifierIC1 (uA741), IC2 (uA741), the diodes D1 D7 (in which D4 and D5 are LED), the photosensitive resistor R1 and R2, the resistor R3 R10, the adjustable resistor RP1, the switch K1, the switch $\mathrm{K} 2$, the relay $\mathrm{J}$ 1, the relay $\mathrm{J}$, the transistor Q1, the transistor Q2 and the DC motor MG1, etc.

The probe head. The probe head is used to track the azimuth angle of the sun, which is composed by the metering tube, the middle partition, the photosensitive resistors R1 and R2, etc ( shown in Figure 3). The photosensitive resistors $\mathrm{R} 1$ and $\mathrm{R} 2$ are divided into two parts by the middle partition, which have been symmetrically installed on both sides of middle partition. The metering tube is covered by the colorless glass and sealed by the glue as the waterproof cover. When the metering tube is facing the sun and the sun lights directly irradiate into the metering tube, the photosensitive resistors R1 and $\mathrm{R} 2$ will receive the equal sun lights. Therefore, the resistance value of photosensitive resistors $\mathrm{R} 1$ and $\mathrm{R} 2$ are the same. Then the output voltage at point $\mathrm{A}$ is $1 / 2 \mathrm{VCC}(6 \mathrm{~V})$ of supply voltage. If the sun lights tend to irradiate the photosensitive resistor $\mathrm{R} 1$, the lights received by photosensitive resistors R1 will be more. However, the photosensitive resistors R2 will receive fewer lights due to the block 
of middle partition and the resistance value of photosensitive resistor $\mathrm{R} 1<$ the resistance value of photosensitive resistor R2. Then the output voltage at point $\mathrm{A}>1 / 2 \mathrm{VCC}$ of supply voltage. Conversely, if the sun lights tend to irradiate the photosensitive resistor R2, the lights received by photosensitive resistors R2 will be more. However, the photosensitive resistors R1 will receive fewer lights due to the block of middle partition and the resistance value of photosensitive resistor $\mathrm{R} 2<$ the resistance value of photosensitive resistor $\mathrm{R} 1$. Then the output voltage at point $\mathrm{A}<1 / 2 \mathrm{VCC}$ of supply voltage. The value of resistor R3 and R4 in circuit should be $33 \mathrm{~K}$ and the value of resistor R5 and R6 should be $200 \mathrm{~K}$. When there is no light irradiation, the dark resistance of photosensitive resistors R 1 and $\mathrm{R} 2$ is still large. In order to ensure the reliability of circuit, the output voltage at point A is still $1 / 2 \mathrm{VCC}$ of supply voltage after decomposing the pressure of resistors R5 and R6 $6^{[4]}$.

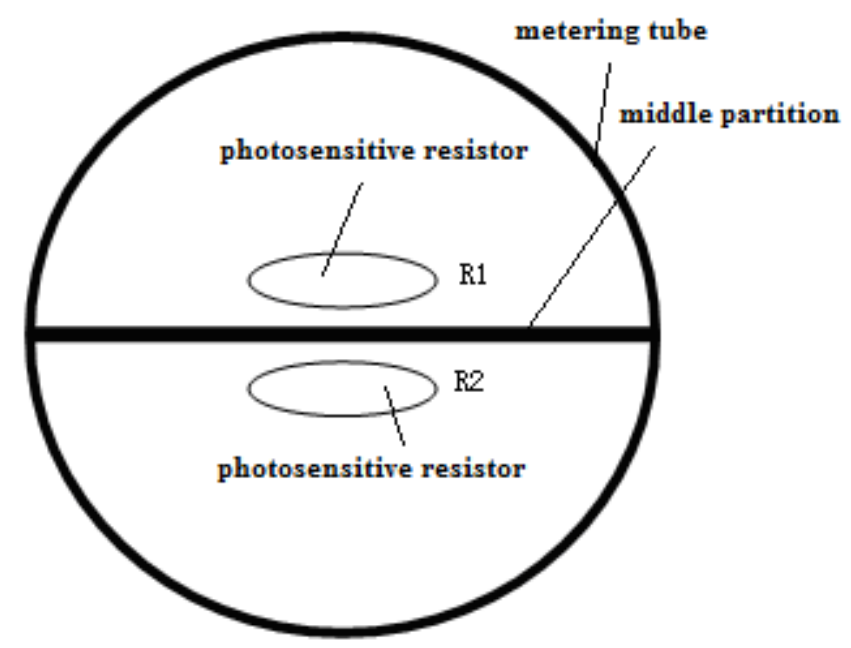

Figure 3 The structure diagram of probe head

The working principle of circuit. The operational amplifier IC1 (uA741) and IC2 (uA741) have formed two voltage comparators with R1 and R2. The reference voltage is $1 / 2$ of $1 / 2 \mathrm{VCC}(+12 \mathrm{~V})$. The photosensitive resistors R1 and R2, the potentiometer RP1 and the resistors R3, R4, R5, R6 and R7 have composed the photosensitive sensor circuit. When R1 and R2 are both affected by the sun lights, the voltage in the center of RP1 and R7 is not changed. If the sun only irradiates R1, the internal resistance of R1 will be decreased, the voltage of (2)pin of IC1 (uA741) will be risen, 6)pin will output the high level, the transistor Q1 will be saturated and turned on, the relay $\mathrm{J} 2$ will be turned on, the changeover contact 3 and contact 1 will be closed and the DC motor MG1 will be positively rotated. If the sun only irradiates $\mathrm{R} 2$, the internal resistance of R1 will be decreased, the voltage of (2) pin of IC2 (uA741) will be risen, (6) pin will output the high level, the transistor Q2 will be saturated and turned on, the relay $\mathrm{J} 1$ will be turned on, the changeover contact 3 and contact 1 will be closed and the DC motor MG1 will be reversely rotated. When the intensities of illumination on the both sides of middle partition are the same, the relay $\mathrm{J} 1$ and $\mathrm{J} 2$ will be turned on and the DC motor MG1 will be stopped. During the constant movement of the sun, the intensities of illumination on the both sides of middle partition will be alternated and the DC motor MG1 will be turned on $\sim$ stopped, turned on $\sim$ stopped, which makes the sun light receiving devices always face the sun.

The adjustable resistor RP1, the diode $\mathrm{D} 1$ and the resistor $\mathrm{R} 7$ provides the reference voltage for the reverse side of IC1 and the positive side of IC2 after decomposing the pressure. As the diode D1 is connected in the circuit, the difference between the reference voltages of IC1 and IC2 will be a silicon $\mathrm{PN}$ junction voltage, which is about $0.6 \mathrm{~V}$. Through adjusting the adjustable resistor RP1, the reference voltage at the "+" side of $\mathrm{IC} 1$ is $6 \mathrm{~V}+(0.2 \mathrm{~V} \sim 0.3 \mathrm{~V})$ and the reference voltage at the "-" side of IC2 is $6 \mathrm{~V}-(0.2 \mathrm{~V} \sim 0.3 \mathrm{~V})$. Therefore, when the output voltage at point $\mathrm{A}$ is greater than $6 \mathrm{~V}+(0.2 \mathrm{~V} \sim$ $0.3 \mathrm{~V})$ or when the output voltage at point $\mathrm{A}$ is less than $6 \mathrm{~V}-(0.2 \mathrm{~V} \sim 0.3 \mathrm{~V})$, the comparator amplifier IC1 or IC2 will output the high level. Otherwise, the comparator amplifier IC1 or IC2 will output the low level. During this period, the $0.6 \mathrm{~V}$ PN junction voltage of diode D1 has provided the fault-tolerant space for the inertia of driving part. 
It is required to send the control signals to the horizontal recognition circuit so as to make the drive system rotate towards the photosensitive resistor $\mathrm{R} 1$ or the photosensitive resistor $\mathrm{R} 2$. In this process, the resistance values of photosensitive resistor R1 and R2 will gradually become equal. The output voltage at point $\mathrm{A}$ will be gradually increased or decreased when the voltage is greater than $6 \mathrm{~V}+$ $(0.2 \mathrm{~V} \sim 0.3 \mathrm{~V})$ or less than $6 \mathrm{~V}-(0.2 \mathrm{~V} \sim 0.3 \mathrm{~V})$. Then the horizontal recognition circuit will be turned on, which will cut off the power of DC motor MG1. However, the drive system will continue to move a slight angle under the influence of inertia force, which is just within the fault-tolerant range of drive system. At the same time, the jitter of the action agencies in the critical position has been eliminated, which has ensured the stable and reliable operation of the whole system. Of course, the size of this fault-tolerant value can be necessarily adjusted according to the circuit and the specific conditions of site.

In order to prevent the accidents, it is required to design the manual control part. When the switch $\mathrm{K} 1$ is pressed, the transistor Q1 will be turned on and the DC motor MG1 will rotate in single direction. When the switch $\mathrm{K} 2$ is pressed, the transistor Q2 will be turned on and the DC motor MG1 will rotate in another direction. Then the manual control will be achieved. During the operation, even if there are some faults, for example, the relays $\mathrm{J} 1$ and $\mathrm{J} 2$ are simultaneously connected and the voltages at the input side and the output side of DC motor MG1 are both higher, the DC motor MG1 will not cause the malfunction and the motor will not be burned out.

D4 is the green LED and D5 is the red LED. As the output display of comparator, they are used for the instructions of driving directions, which is helpful to adjust the circuit and observe the operation status of system at any time. D2 and D3 have played an isolated role in the circuit, which prevents the high level from affecting the low-level signals output by IC1 and IC2 when the manual switch is pressed. $\mathrm{J} 1$ and $\mathrm{J} 2$ are the single-pole double-throw relay and the contact capacity can be selected by the operating current of DC motor MG1. The SPDT switch contacts of J1 and J2 are used to control the positive and reverse rotation of DC motor MG1. D6 and D7 are the relay protection diodes. As for this system, it is not needed to worry about whether it will automatically return in next morning. When the sun rises, the light intensities on the both sides of middle partition can't be exactly equal. Then the control circuit of this design will control the DC motor MG1 so as to drive the receiving device to rotate from west to east until the sun light receiving device faces the sun.

\section{The installation and commissioning of system}

It is required to consider the mechanical adjustment mechanism and the circuit board as a system for debugging. The machinery sectors and the circuit board should be fixed on the bracket for debugging. The DC motor MG1 should not be given power at first. It is necessary to use the multi-meter to measure the voltages at the "+" side of IC1 and the"-"side of IC2 when the light intensities of photosensitive resistors $\mathrm{R} 1$ and $\mathrm{R} 2$ are the same. At the same time, it is also required to adjust the resistance value of RP1 so as to make them equal. The flashlight is regarded as the light source, which will be placed in a position with a distance of $40 \mathrm{~cm} \sim 3 \mathrm{~m}$ from the probe head. D4 and D5 should be lighted up one after another. Afterwards, the DC motor MG1 will be turned on and the system will automatically search for the light source under the drive of motor.

\section{Conclusion}

In this paper, the designed photoelectric solar tracking system is simple and reliable. The cost is low and the operation is sensitive and prompt. The automatic tracking can be achieved and the manual operation control can be also realized. At the same time, the intelligent tracking can be realized through combining with the single-chip control system and GPS. It is suitable for the solar tracking of various solar energy utilization devices such as the medium and small solar light collection systems, the solar power systems, the solar heating systems and some others. 


\section{Acknowledgment}

Project Funds: The Regional Economic Research in Zunyi Normal College in 2010 (ZE201006), the key supporting disciplines in Guizhou Province (issued by the department of higher education in Guizhou, [2011] No. 275)

\section{References}

[1] Gao Fei, Zhu Changhan, Lin Ruoci. New Energy and its Application in Architectural Lighting[J]. China Illuminating Engineering Journal, 2010 (6) : 32-42.

[2] Xiao Huaichun. Application of GPS in sun tracking system[D]. Beijing: Beijing Jiaotong University, 2009.1-66.

[3] Li Min. Study on the key technologies of sun lighting[D]. Chongqing: Chongqing University, 2008.1-76.

[4] Xie Biao. Practical solar tracking circuit[J]. Electronic world, 2009 (11) : 40-41. 